\title{
Nanoparticles generated by laser in liquids as contrast medium and radiotherapy intensifiers
}

\author{
Nancy Restuccia $^{1}$ and Lorenzo Torrisi ${ }^{1}$ \\ ${ }^{1}$ Dottorato di Ricerca in Fisica, Dip.to di Scienze Fisiche-MIFT, V.le F. S. D'Alcontres 31, 98166 S. Agata (ME), Italy
}

\begin{abstract}
The synthesis of $\mathrm{Au}$ and $\mathrm{Ag}$ nanoparticles (NP) though laser ablation in liquids as a function the laser parameters is presented. Spherical NPs with diameter distribution within 1 and $100 \mathrm{~nm}$ were prepared by laser ablation in water. The nanoparticles characterization was performed using optical spectroscopy and electronic microscopy (SEM and TEM) measurements. Studies of the possible use of metallic nanoparticles as intensifier of diagnostics imaging contrast medium and absorbing dose from ionizing radiations in traditional radiotherapy and protontherapy are presented. Examples of in vitro (in tissue equivalent materials) and in vivo (in mice), were conducted thank to simulation programs permitting to evaluate the enhancement of efficiency in imaging and therapy as a function of the NPs concentrations and irradiation conditions.
\end{abstract}

\section{Introduction}

In recent years, technological development has allowed us to prepare, manipulate and characterize, more nanosized materials. Metallic nanoparticles (NP), based on $\mathrm{Ti}, \mathrm{Au}, \mathrm{Ag}, \mathrm{Bi}$ and others, have numerous fields of applications: from electronics (detectors, solar cells, optoelectronics devices, sensors, nanoacoustic) to telecommunications (nonlinear optics, nanophotonics, nanothermic), biology and medicine (photothermal therapy, radiation and biological imaging diagnostics) and physical-chemistry (catalysts, preparing targets).

Metallic nanoparticles embedded in an insulating medium behave like ionizing radiation absorbing centres, being able to show certain absorption resonances bands at specific wavelengths, thank to the Surface Plasmon Resonance (SPR) absorption effect [1]. Their size distribution in a fluid changes the electronic and mass density and the equivalent atomic number of the material. The choice of gold as a starting material to prepare nanoparticles is not accidental. Gold, in fact, is stable, not oxidable and biocompatible for humans and, more in general, also for other living beings. In the light of these results, the medicine demonstrates a particular interest to the gold and silver nanoparticles for different aspects concerning prosthesis, interface adhesion, contrast medium for X-ray images and high Z-element for high absorbent radiations.

In the present study it was determined experimentally the behaviour of $\mathrm{Au}$ and $\mathrm{Ag}$ nanoparticles (Au NP and $\mathrm{Ag}$ $\mathrm{NP}$ ) in therapy and in diagnostics, in particular by calculating the ratio of the dose delivered to a given depth and the dose delivered to the surface in various media (water, adipose tissue and compact bone) in presence or not of the nanoparticles. The anticancer potential of the gold and silver nanoparticles is generated by the different physical-chemical properties advantages. Numerous studies have demonstrated the safety and biocompatibility of $\mathrm{Au}$ and $\mathrm{Ag}$ both in vitro and in vivo, suggesting that the gold nanoparticles can be administered safely with minimal inflammatory activation and few local or systemic side effects [2-4] enhancing the equivalent $\mathrm{Z}$-value of the treated tissues at significant nanoparticle concentrations.

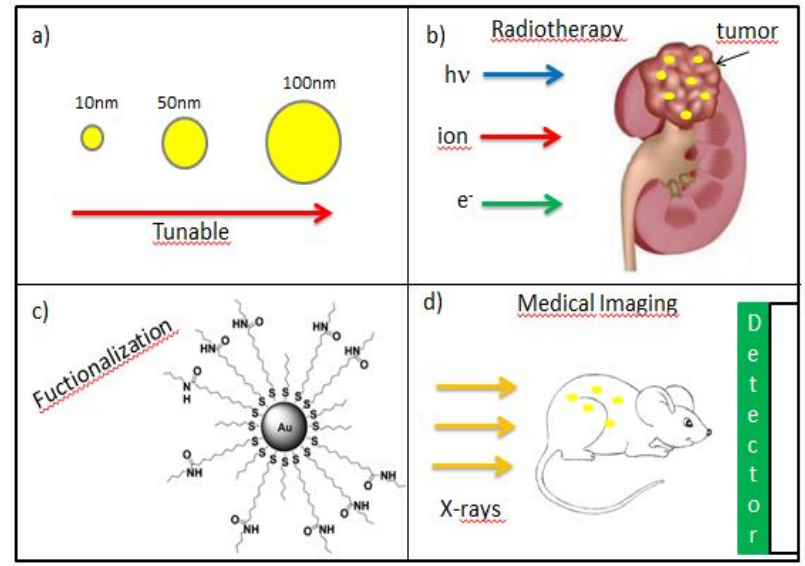

Fig. 1. Au NP with various shapes and size (a) and biofunctionalized with various biomolecules (c). The NP have the ability to enhance radiation therapy of tumours (b), as well as high-Z imaging contrast agents (d).

Fig. 1 shows Au NP of various shapes and sizes (a), their functionalization with particular molecules which allow the transfer to the tumour sites via biological processes (c) or use of drugs or by localized injection into the tumour zone. The NP so attached to the desease tissues

* Corresponding author: 1torrisi@unime.it 
have the ability to improve radiotherapy efficiency (by $\mathrm{X}$-rays, ions and electron beams) (b), and the imaging contrast of localized tissues (d).

The high atomic number of gold $(Z=79)$ allows a high absorption and enhancement of ionizing radiation, as well as the X-ray attenuation and X-ray characteristic line fluorescence emission for higher imaging contrast applications [5]. Moreover, Au NP permit to enhance the electron and ion stopping power increasing the local release of ionizing energy in the cells enriched of $\mathrm{Au}$ _NP fluids.

\section{Materials and Methods}

Laser ablation of metal in water describes a process in which atoms are removed from a solid surface by the irradiation of a high-intensity laser beam and are dispersed in the liquid where aggregate as nanoparticles. Pulsed laser ablation in a liquid medium is a method used to produce a clean, stable, and relatively homogeneous dispersion of nanoparticles in a liquid. Our experimental set-up consists of a pulsed laser, Nd:Yag, operating at fundamental frequency $(1064 \mathrm{~nm})$ with a pulse of $200 \mathrm{~mJ}$ energy and $3 \mathrm{~ns}$ duration, a pulse repetition rate of $10 \mathrm{~Hz}$, and a set of focusing optics. The Fig. 2 shows the scheme (a) and a photo (b) of the experimental set up used to prepare the liquid solution containing the Au NPs.
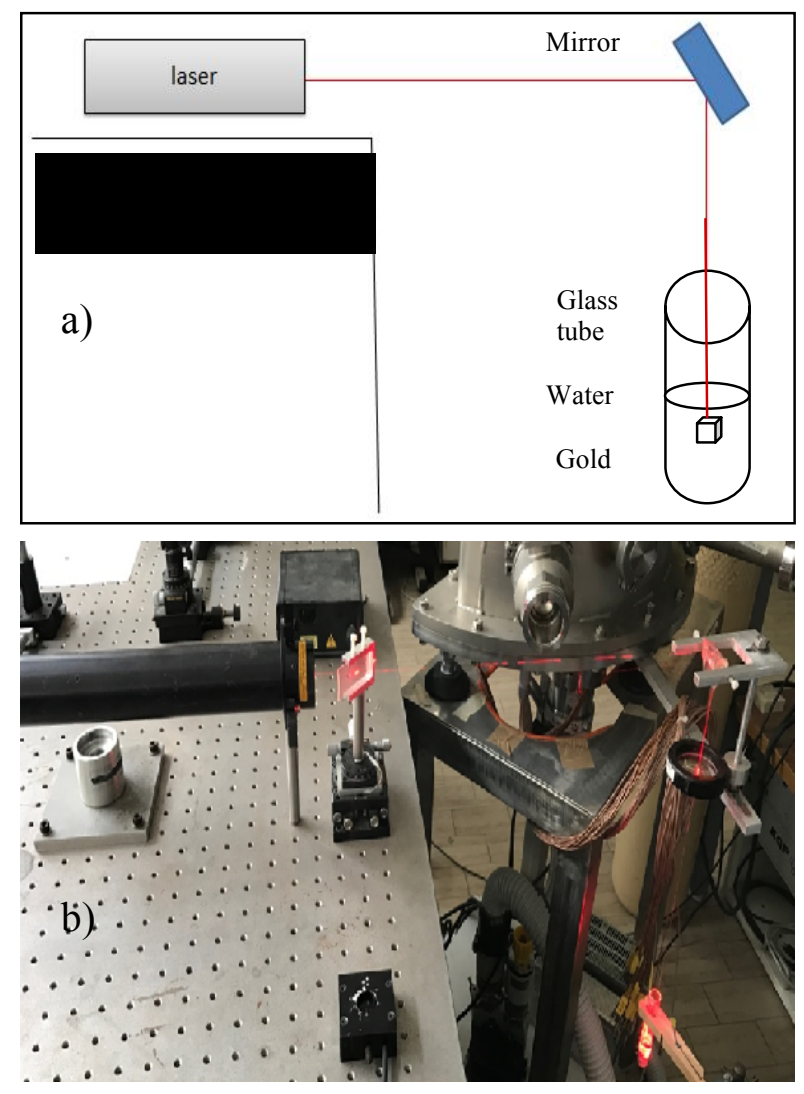

Fig. 2 . Laser ablation in liquid set up scheme (a) and photo of set up in our laboratory (b).
The Fig. 3 shows the prepared solution at 0 minutes, 5 minutes and 20 minutes of $\mathrm{Au}$ laser ablation in water. The solution changes its color with the irradiation time and assumes from a mild pink color up to a red color with the NP concentration increment. As the nanoparticles are released from the sample, the solution takes on a different color depending on the size of the nanoparticles, shape and nature of the liquid.

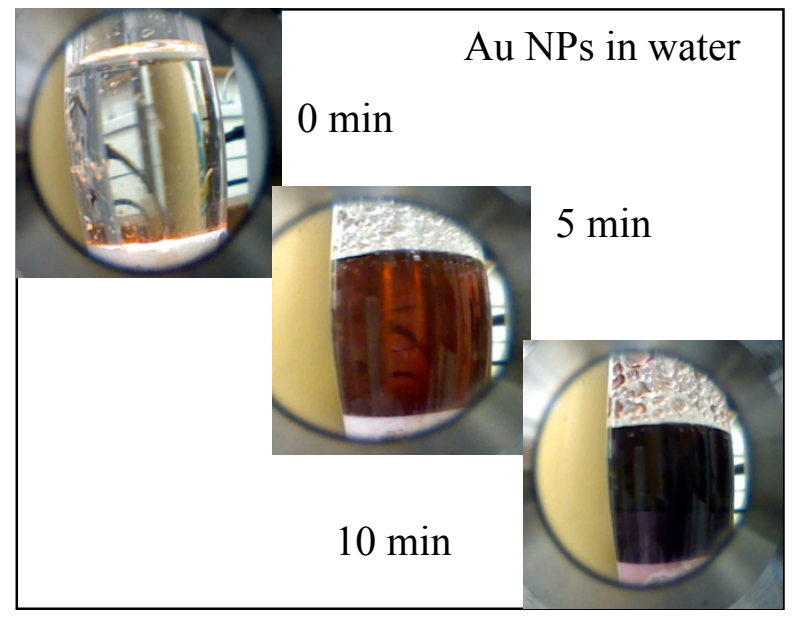

Fig. 3. Solution of $\mathrm{Au} \mathrm{Np}$ in glass tube produced by laser ablation at different times.

A new compact X-ray imaging system emitting photons at a maximum energy of $45 \mathrm{keV}$ with different exposition times, recording the images on a fast CCD digital recorder (Bruker-In vivo imaging system MS-FX PRO) has been employed in our study.

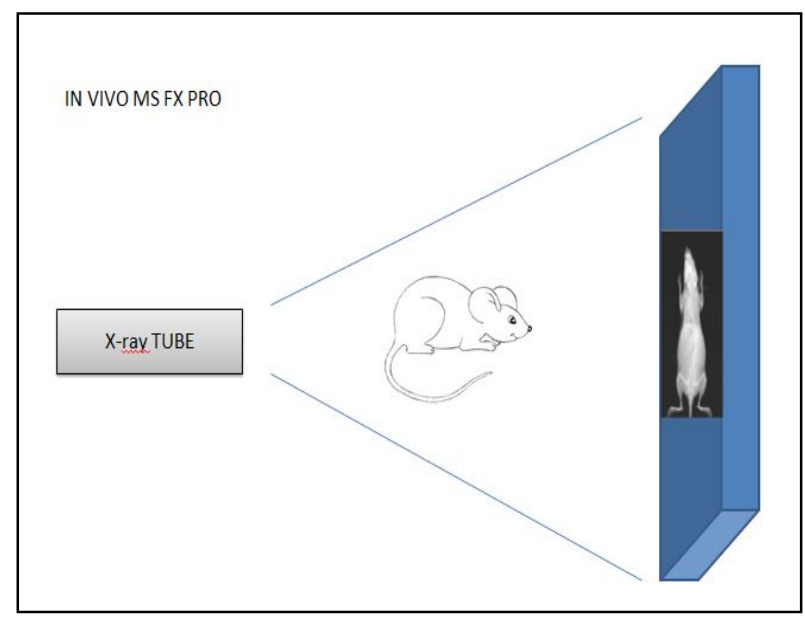

Fig. 4. Scheme of the X-rays imaging system.

The system uses X-rays at energy selectable between 20 and $45 \mathrm{keV}$, a maximum electron current of $500 \mathrm{~mA}$, spot diameter of $60 \mathrm{~mm}$, with a beryllium window filter. The exposition time can be in single capture of $1.2 \mathrm{~s}$, or in multiple captures overlapping up to 32 image captures. Fig. 4 shows a scheme of the X-ray absorption image used in our laboratory [6-9].

The output image is typically grayscale and may be obtained thought of as a shadow of the object in the path of the X-ray beam. In a classic X-ray image with film, areas that appear white represents dense tissues (like 
bone or inflammation and infected lung tissue that can obstruct the passage of X-ray energy). Meanwhile, tissue that is not dense (like the lungs, which are filled with air) will have contrast at the opposite end of the grayscale spectrum and appear black. The new used X-ray image system records images on a CCD detector though different filters, such as beryllium, aluminum and other thin films, in order to improve the signal-to-noise ratio. This tool has allowed us to carry out animal measurements to verify the effectiveness of high- $Z$ metallic nanoparticles as a potential contrast medium injectable by intravenous, peritoneal or ingestion way. It permits to record X-ray images not only using transmitted X-rays but also using secondary X-ray characteristic fluorescence emission lines, as obtained using Au and Ag NPs.

\section{Results}

The SEM images of the solution containing Au NP, deposited as a drop on a Si substrate and dried at $50{ }^{\circ} \mathrm{C}$, indicate the presence of spherical shapes with a narrow size distribution, with about $100 \mathrm{~nm}$ in diameter. The optical properties of the spherical gold nanoparticles are highly dependent on the nanoparticle diameter, shape and nature of the used liquid. Smaller Au nanospheres primarily absorb light near $520 \mathrm{~nm}$, while larger spheres exhibit increased scattering and have absorption bands that broaden significantly and shift towards longer wavelengths (phenomenon known as red-shifting).

Fig. 5 reports the SEM images of Au NPs at low magnification (a) and high magnification (b) deposited on a silicon substrate as a liquid drop.

Larger spheres scatter more light both because they have larger optical cross sections, and also because their albedo (a ratio of scattering to total extinction) increases with size. The optical properties of the gold and silver nanoparticles change when particles aggregate and the conduction electrons near each particle surface become delocalized and are shared amongst neighboring particles. The gold nanoparticles absorb and scatter visible light with extraordinary efficiency. The absorbance is however restricted to a specific band of wavelengths. It depends on the size of NP, on the shape and on the surrounding medium, as reported in literature [1]. Their strong interaction with light occurs because the conduction electrons on the metal surface undergo collective oscillation when excited by light at specific wavelengths. This oscillation is known as a surface plasmon resonance (SPR), and causes the absorption and scattering of visible light or near UV range or in the IR. When this occurs, the surface plasmon resonance shifts to lower energies, causing the absorption and scattering peaks to red-shift to longer light wavelengths. UVVisible spectroscopy can be used as a simple and reliable method for monitoring the stability of nanoparticle solutions.

The optical properties of spherical gold nanoparticles are highly dependent on the diameter of the nanoparticles.

Fig. 6 shows the effect of light illumination of metallic nanoparticles which becomes electric dipoles induced by the incident light with an electric field opposite to the incident one producing light attenuation [10].
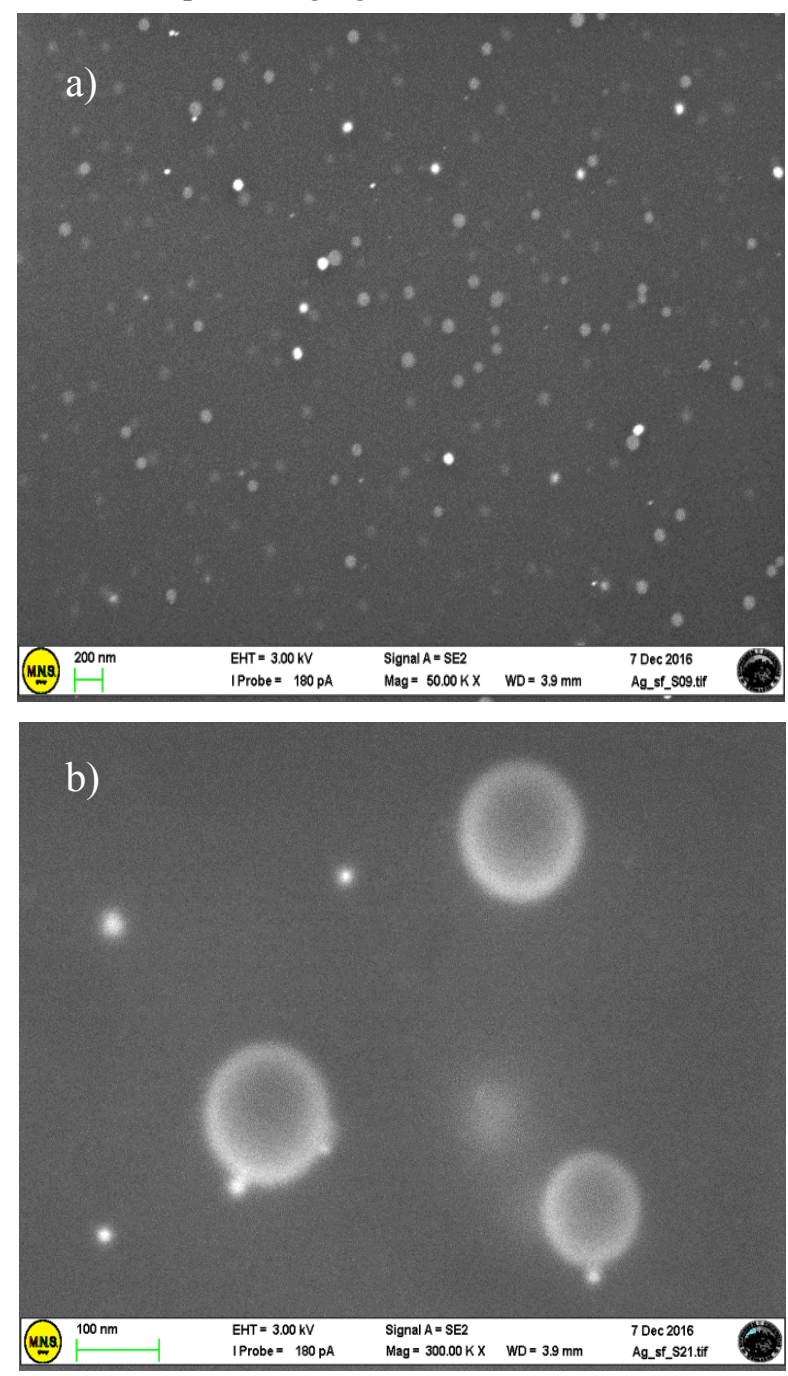

Fig. 5. SEM image of gold nanoparticles.

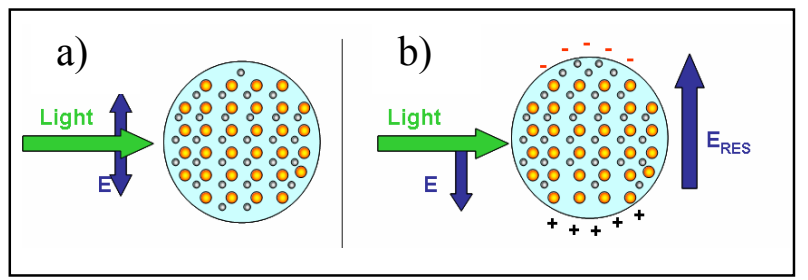

Fig. 6. Dipole induced by incident light on metallic nanoparticles and generation of opposite dipole electric field producing light attenuation.

Fig. 7 shows the result of the optical characterization of the Au NPs in water which absorbance can be measured as a function of the wavelength in order to evidence the SPR absorption bands typical of the prepared solution. Fig. 7a reports the absorbance as a function of the wavelength and of the diameter of the spherical Au NPs in water. By increasing the diameter, the absorption band shift towards higher wavelengths. Fig. $7 \mathrm{~b}$ shows the different absorbance in presence of separated nanoparticles and of aggregated nanoparticles, up to 1 micron in size, in water. As the particles destabilize, the 
original extinction peak will decrease in intensity (due to the depletion of stable nanoparticles), and often the peak will broaden or a secondary peak will form at longer wavelengths (due to the formation of aggregates) and shifted towards high wavelengths.
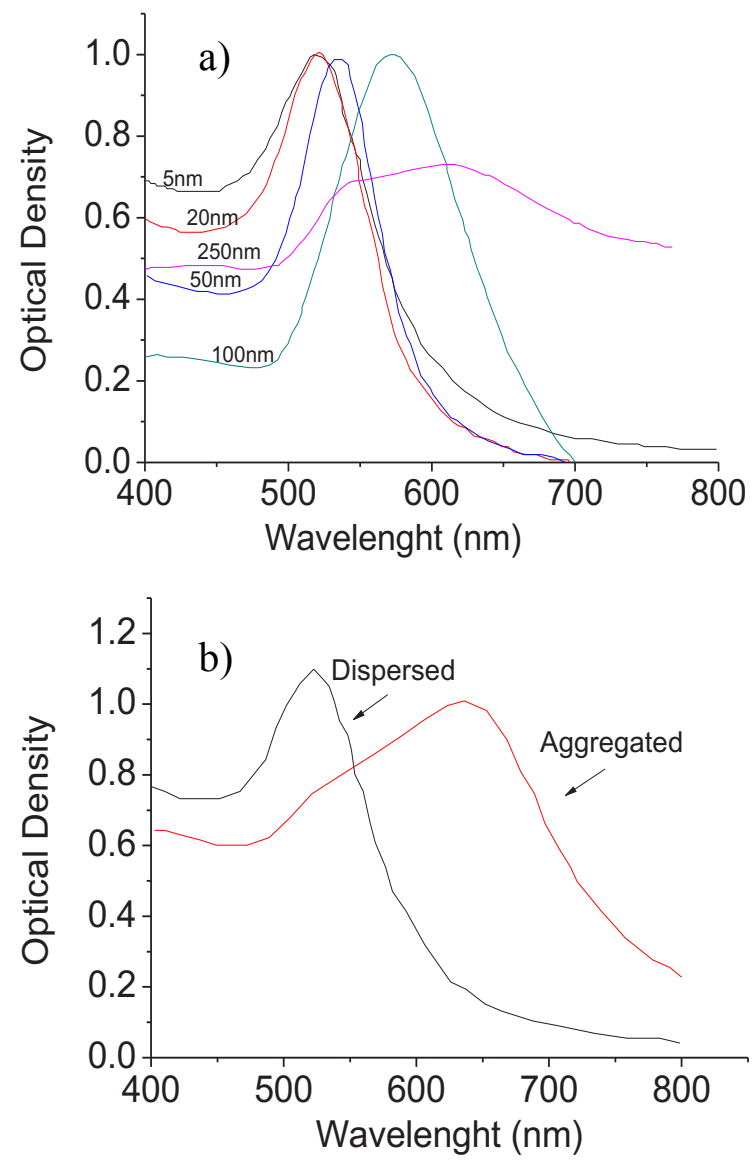

Fig. 7. UV-Vis spectrum of gold nanoparticles of different sizes (a) and extinction spectra of dispersed and agglomerated gold nanoparticles (b).

In order to avoid the nanoparticle aggregation phenomenon, in water was added a biocompatible solution of $1 \mu \mathrm{g} / \mathrm{ml}$ of sodium citrate that permits their uniform dispersion minimizing the collateral effect of coalescence [11-13].

Water solutions containing gold and silver nanoparticles, both generated by ablation in water and analyzed through SEM and TEM microscopy, giving an average diameter of about $50 \mathrm{~nm}$, were injected in healthy mice. The administration of $0.01 \mathrm{ml}$ solution containing nanoparticles per gram of mouse weight was given through intraperitoneal way. The Fig. 8 shows the differences between the X-rays of fluorescence images obtained using gold and silver nanoparticles injected in mice. Once injected gold nanoparticles are quickly absorbed and after about 120 minutes they are deposited in the colon (middle images), after 24 hours there is a remainder accumulated in the livers (bottom images). Instead, measurements demonstrated that silver nanoparticles are absorbed more slowly and the first visible results are found after 24 hours with a significant build up in the colon of the mouse.

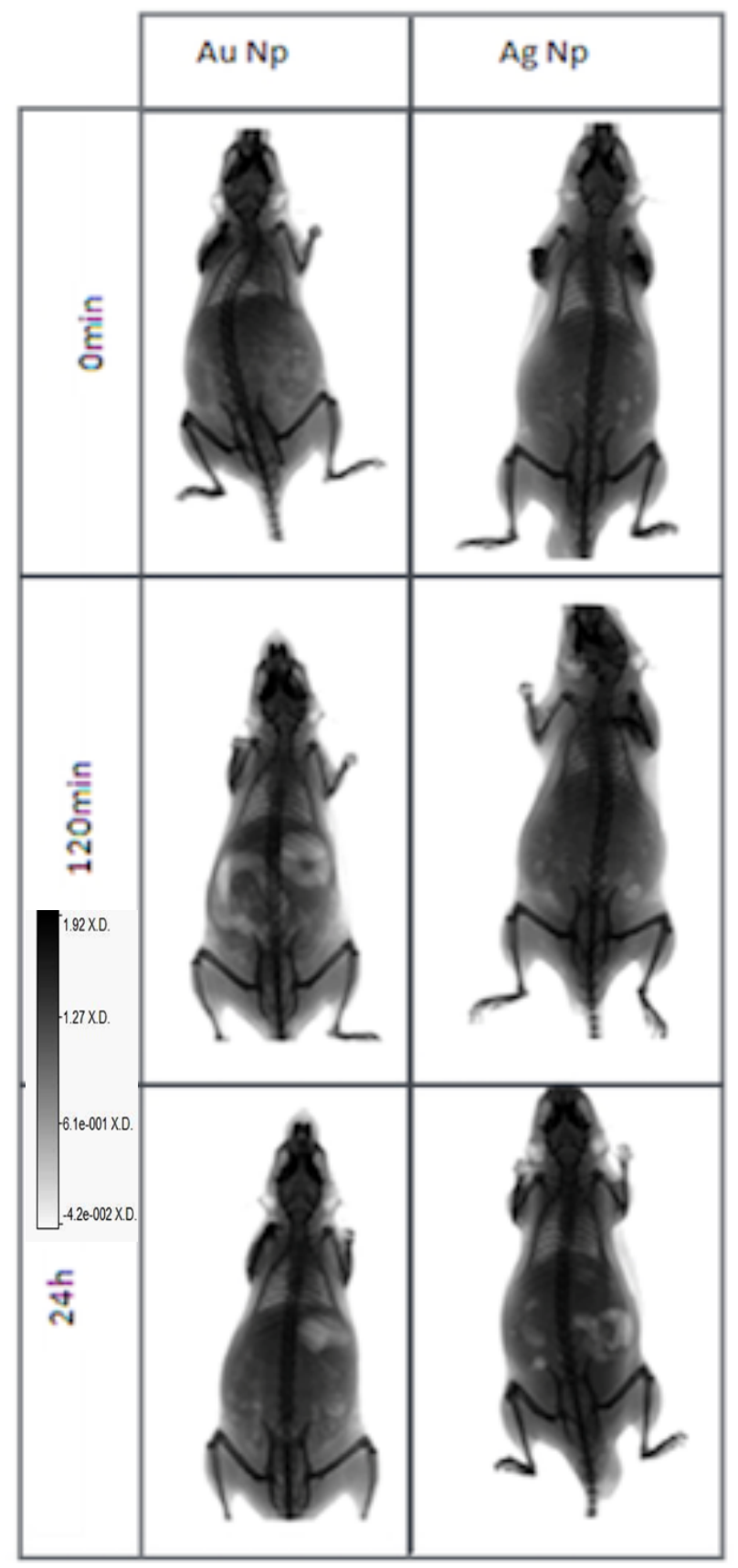

Fig. 8. X-rays fluorescence images of $\mathrm{Au}$ and $\mathrm{Ag}$ in mice as a function of the time from the peritoneal injection.

This result leads us to conclude that blood circulation of silver nanoparticles produces a slower up-take than that relative to gold nanoparticles. Thus the two kind of nanoparticles have a different metabolic up-take effect. The high ionizing radiation absorption of high $\mathrm{Z}$ metal nanoparticles, such as gold, can be used to improve traditional radiotherapy with photons and electrons and the innovative one using protons and carbon ions. By inserting high- $Z$ NPs into the site of the tumor it is possible to increase the dose released on that particular site. To this aim, theoretical studies have been carried out and are in progress. 
For example, for $100 \mathrm{MeV}$ protons in the soft tissue, the stopping powers at $3 \mathrm{~cm}$ deep $\left(\mathrm{S}_{\text {prof }}\right)$ and at surface $\left(\mathrm{S}_{\text {sup }}\right)$, can be calculated using NIST database and SRIM Code [14-15]. Their ratio $\left(\mathrm{R}_{\text {tissue }}\right)$ is increased by a factor $7 \%$ when $\mathrm{Au}$ NPs are added, at a concentration of 100 $\mathrm{mg} / \mathrm{ml}$, at $3 \mathrm{~cm}$ depth where the tumor is localized, as described by the comparison of the equations (1) and (2) reported in the following.

$100 \mathrm{MeV}$ protons:

$R_{\text {tissue }}=\frac{S_{\text {prof }}}{S_{\text {sup }}}=\frac{605,3}{7,495}=80,76$

$R_{\text {tissue }+A u}=\frac{S_{\text {prof tissue }+A u}}{S_{\text {sup tissue }}}=\frac{647,24}{7,495}=86,36$

Another example is evaluated for $100 \mathrm{keV}$ photons in soft tissue with the tumor located at $3 \mathrm{~cm}$ deep and with $1 \mathrm{~cm}$ tumor thickness. The Lambert-Beer formula is used to calculate the transmitted intensity $\left(\mathrm{I}_{\mathrm{T}}\right)$ of the incident one $\left(\mathrm{I}_{0}\right)$ in the tissue thickness $\Delta \mathrm{x}$ on the base of the energy massive attenuation coefficient $\left(\mu_{\mathrm{e}}\right)$ and density ( $\rho)$. By adding Au nanoparticles to the tumor site, the transmitted intensity decreases indicating an increment of the absorbed radiation dose. The calculations indicate a dose increment of about $85 \%$ with the use of Au NPs at $100 \mathrm{mg} / \mathrm{ml}$ concentration, as reported in the calculations of the equations (3) and (4).

$100 \mathrm{keV}$ photons:

$I_{T}=I_{0} \cdot e^{-\frac{\mu_{e}}{\rho} \cdot \rho \cdot \Delta x}=84,14 \cdot e^{-0,169 \cdot 1,02 \cdot 1}=70,79 \mathrm{keV}$

$I_{T+A u}=I_{0} \cdot e^{-\frac{u_{n}}{\rho} \cdot \rho \cdot \Delta x}=70,79 \cdot e^{-0,68 \cdot 2,85 \cdot 1}=10,56 \mathrm{keV}$

Here the results are reported quantitatively; we immediately notice that thanks to the use of gold nanoparticles, radiotherapy becomes much more effective because the whole dose of radiation should be directly given to the tumor site and not to the surrounding healthy tissue. Reported results are in agreement with literature data $[2,3,6,7]$.

\section{Conclusions}

The use of high- $Z$ metallic nanoparticles in the field of radiotherapy and diagnostics promises great results. It improves imaging quality as contrast medium and increases the efficiency of radiotherapy by localizing the released dose to the nanoparticles at tumor site. Actual experiments on mice are performed thank to the collaboration with biologists following the work scheme reported in Fig. 1.

This work was supported by the "Research and Mobility" project of Messina University No. 74893496, scientifically coordinated by Professor L. Torrisi.

\section{References}

1. M. A. Garcia "Surface Plasmon in metallic nanoparticles: Fundamental and applications." Journal of Physics: Applied Physics 44, 28 (2011).

2. F. Jay et al. "Gold nanoparticles in radiation research: potential applications for imaging and radiosensitization" Transl Cancer Res 2013;2(4):280-291.

3. Khlebtsov N, Dykman L. Biodistribution and toxicity of engineered gold nanoparticles: a review of in vitro and in vivo studies. Chem Soc Rev. 2011;40:1647-71.

4. Connor EE, Mwamuka J, Gole A, et al. Gold nanoparticles are taken up by human cells but do not cause acute cytotoxicity. Small. 2005;1:325-7.

5. L. Torrisi, et al."Effect of metallic nanoparticles in thin foils for laser ion acceleration" Phys. Scripta 9 (2015).

6. D. R. Cooper et al "Gold nanoparticles and their alternatives for radiation therapy enhancement" Frontiers in Chemistry (2014).

7. Vega and U.O. Häfeli "Utilization of nanoparticles as X-ray contrast agents for diagnostic imaging applications", Contrast Media Mol. Imaging 10 (2015).

8. J. Zou et al., "Micro CT visualization of silver nanoparticles in the middle and inner ear of rat and transportation pathway after transtympanic injection", Journal of Nanobiotechnology (2015).

9. K. Saha, S.S. Agasti, C. Kim, X. Li, and V.M. Rotello, Gold Nanoparticles in Chemical and Biological Sensing, Chem Rev. 112(5), 2739-2779 (2012).

10. S.K. Ghosh and T. Pal, "Interparticle Coupling Effect on the Surface Plasmon Resonance of Gold Nanoparticles": From Theory to applications, Chem. Rev. 107, 4797-4862 (2007).

11. L. Torrisi , "Radiotherapy Improvements by Using $\mathrm{Au}$ Nanoparticles", Recent Patents on Nanotechnology, 9(2), 114-125 (2015).

12. L. Torrisi N. Restuccia, I. Paterniti "Gold Nanoparticles produced by laser ablation in liquids for improvements of diagnostic imaging”, Recent Patents on Nanotechnology, (2017).

13. L. Torrisi, N. Restuccia, S. Cuzzocrea, I. Paterniti, I. Ielo, S. Pergolizzi, M. Cutroneo and L. Kovacik, "Laser-produced Au nanoparticles as X-ray contrast agents for diagnostic imaging”, Gold Bullettin 50(1) pp 51-60 (2017).

14. J. Ziegler, SRIM, The Stopping and Range of Ions in Matter, actual website 2017: http://www.srim.org/

15. NIST database 2017 https://www.nist.gov, J. H. Hubbell and S. M. Seltzer Radiation Physics Division, PML. 